\title{
Classificação do pré-natal em maternidades do Espírito Santo conforme recomendações da Organização Mundial da Saúde e do Ministério da Saúde do
}

\section{Brasil}

\author{
Classification of prenatal care in maternity hospitals in Espírito Santo according to the \\ recommendations of the World Health Organization and Brazilian Ministry of Health \\ Clasificación de la atención prenatal en las maternidades de Espírito Santo según las \\ recomendaciones de la Organización Mundial de la Salud y el Ministerio de Salud de Brasil
}

Recebido: 08/09/2021 | Revisado: 16/09/2021 | Aceito: 20/09/2021 | Publicado: 21/09/2021

Katrini Guidolini Martinelli ORCID: https://orcid.org/0000-0003-0894-3241 Programa de Pós-graduação em Saúde Coletiva, Brasil E-mail: katrigm@gmail.com Silvana Granado Nogueira da Gama ORCID: https://orcid.org/0000-0002-9200-0387 Fundação Oswaldo Cruz, Brasil E-mail: silvana.granado@gmail.com Érica Marvila Garcia ORCID: https://orcid.org/0000-0001-9477-2383 Universidade de São Paulo, Brasil E-mail: ericamarvila@usp.br

Edson Theodoro dos Santos-Neto ORCID: https://orcid.org/0000-0002-7351-7719 Universidade Federal do Espírito Santo, Brasil E-mail: edsontheodoro@uol.com.br

\begin{abstract}
Resumo
Objetivo: Classificar o atendimento pré-natal de mulheres atendidas para o parto em maternidades do Espírito Santo, por meio de um índice universal, envolvendo as recomendações da Organização Mundial da Saúde (OMS), e um índice nacional, do Ministério da Saúde do Brasil (MS), além de analisar os fatores associados à inadequação do prénatal. Métodos: Estudo transversal com puérperas participantes do estudo "Nascer no Brasil" em maternidades do Espírito Santo, entre 2011-2012. Realizou-se regressão logística multivariada para verificar os fatores associados à inadequação do pré-natal. Resultados: 450 puérperas participaram do estudo. Em mais da metade dos itens que compõem o protocolo da OMS obteve-se $80 \%$ ou mais de execução. A suplementação de ferro e ácido fólico foram os itens menos executados (20\%). Com base nas recomendações do MS, os procedimentos clínico-obstétricos foram os mais executados, enquanto o exame citopatológico de colo uterino foi o menos registrado (9,3\%). Mais de 50\% das puérperas tiveram pré-natal considerado inadequado. Os fatores associados à inadequação do pré-natal foram: não ter trabalho remunerado $(\mathrm{OR}=2,09 ; \mathrm{IC} 95 \%=1,22-3,57)$, não ter intenção de engravidar $(\mathrm{OR}=1,91 \mathrm{e} \quad \mathrm{OR}=1,88$; IC95\%=1,08-3,37), residir fora da capital do estado $(\mathrm{OR}=1,87$ e OR=1,91; IC95\%=1,11-3,17), pré-natal no setor público $(\mathrm{OR}=4,92$; IC95\%=1,65-14,68) e ser multípara $(\mathrm{OR}=9,85$; IC95\%=2,92-33,14). Conclusão: Mulheres mais vulnerabilizadas foram as que mais tiveram inadequação do pré-natal, o que demanda mudanças no modo de captação e adesão das gestantes ao atendimento pré-natal, ampliando o papel social dos serviços públicos de saúde, com base em protocolos pré-estabelecidos, além de ampliar o acesso ao planejamento reprodutivo.

Palavras-chave: Cuidado pré-natal; Avaliação de resultados em cuidados de saúde; Mecanismos de avaliação da assistência à saúde; Serviços de saúde materno-infantil.

\footnotetext{
Abstract

Objective: to classify the prenatal care of women attended for childbirth in maternity hospitals in Espírito Santo, through a universal index, involving the recommendations of the World Health Organization (WHO), and a national index, from the Ministry of Health of Brazil (MHB), in addition to analyzing the factors associated with inadequate prenatal care. Methods: Cross-sectional study with postpartum women participating in the study "Born in Brazil" in maternity hospitals in Espírito Santo, between 2011-2012. Multivariate logistic regression was performed to verify the factors associated with inadequate prenatal care. Results: 450 postpartum women participated in the study. In more than half of the items that make up the WHO protocol, $80 \%$ or more was achieved. Iron and folic acid
} 
supplementation were the least performed items (20\%). Based on the recommendations of the MHB, clinical-obstetric procedures were the most performed, while the uterine cervix cytopathological examination was the least recorded (9.3\%). More than $50 \%$ of mothers had prenatal care considered inadequate. The factors associated with inadequate prenatal care were: not having a paid job $(\mathrm{OR}=2.09 ; 95 \% \mathrm{CI}=1.22-3.57)$, not having an intention to become pregnant $(\mathrm{OR}=1.91$ and $\mathrm{OR}=1.88 ; 95 \% \mathrm{CI}=1.08-3.37)$, living outside the state capital $(\mathrm{OR}=1.87$ and $\mathrm{OR}=1.91 ; 95 \% \mathrm{CI}=1.11-$ $3.17)$, prenatal care in the public sector $(\mathrm{OR}=4.92 ; 95 \% \mathrm{CI}=1.65-14.68)$ and being multipara $(\mathrm{OR}=9.85 ; 95 \% \mathrm{CI}=2.92-$ 33.14). Conclusion: The most vulnerable women were the ones who had the most inadequate prenatal care, which requires changes in the way pregnant women are recruited and adhered to prenatal care, expanding the social role of public health services, based on predetermined prenatal protocols, in addition to expanding access to reproductive planning.

Keywords: Prenatal care; Outcome assessment, health care; Health care evaluation mechanisms; Maternal-child health services.

\section{Resumen}

Objetivo: Clasificar la atención prenatal de mujeres atendidas por parto en maternidades de Espírito Santo, a través de un índice universal, que involucra las recomendaciones de la Organización Mundial de la Salud (OMS), y un índice nacional, del Ministerio de Salud de Brasil (MS), además de analizar los factores asociados a una atención prenatal inadecuada. Métodos: Estudio transversal con puérperas participantes del estudio "Nacidas en Brasil" en maternidades de Espírito Santo, entre 2011-2012. Se realizó una regresión logística multivariante para verificar los factores asociados con una atención prenatal inadecuada. Resultados: 450 mujeres en posparto participaron en el estudio. En más de la mitad de los ítems que componen el protocolo de la OMS se logró el 80\% o más. La suplementación con hierro y ácido fólico fueron los ítems menos realizados (20\%). De acuerdo con las recomendaciones del Ministerio de Salud, los procedimientos clínico-obstétricos fueron los más realizados, mientras que el examen citopatológico del cuello uterino fue el menos registrado (9,3\%). Más del 50\% de las madres tenían una atención prenatal considerada inadecuada. Los factores asociados con una atención prenatal inadecuada fueron: no tener un trabajo remunerado (OR $=2,09 ; \mathrm{IC} 95 \%=1,22-3,57)$, no tener la intención de quedar embarazada $(\mathrm{OR}=1,91$ y OR=1,88; IC95\% =1,08-3,37), vivir fuera de la capital del estado $(\mathrm{OR}=1,87$ y $\mathrm{OR}=1,91$; IC 95\% =1,11-3,17), atención prenatal en el sector público $(\mathrm{OR}=4,92$; IC 95\% =1,65-14,68) y ser multípara $(\mathrm{OR}=9,85 ;$ IC95\% = 2,92-33,14). Conclusión: Las mujeres más vulnerables fueron las que tuvieron una atención prenatal más inadecuada, lo que requiere cambios en la forma en que las embarazadas son reclutadas y adheridas a la atención prenatal, ampliando el rol social de los servicios de salud pública, con base en los protocolos prenatales establecidos, en además de ampliar el acceso a la planificación reproductiva.

Palabras clave: Atención prenatal; Evaluación de resultado en la atención de salud; Mecanismos de evaluación de la atención de salud; Servicios de salud materno-infantil.

\section{Introdução}

A avaliação de programas e políticas de saúde - que tem ganhado destaque no Brasil - é uma ferramenta relevante para o planejamento e para a gestão dos serviços de saúde (Polgliane et al., 2014). Seus resultados podem subsidiar a manutenção ou a modificação das estratégias adotadas no cuidado pré-natal com o objetivo de melhorar a qualidade da assistência prestada (Silva et al., 2019).

Estudos que analisam a assistência pré-natal consideram, mais frequentemente, a idade gestacional ao iniciar o acompanhamento e o número de consultas pré-natal realizadas (Heaman et al., 2019; Nussey et al., 2020; Osterman \& Joyce A. Martin, 2018; Santos Neto et al., 2013; Vale et al., 2021). Entretanto outros critérios devem ser considerados na avaliação da qualidade da assistência prestada à gestante (Yeoh et al., 2018), considerando que a adesão ao pré-natal no primeiro trimestre e a realização de seis ou mais consultas tem aumentado bastante no Brasil (DATASUS, 2021). O incremento no número das consultas, unicamente, não tem refletido na queda da razão de morte materna (55/100.000 nascidos vivos) e da taxa de mortalidade neonatal precoce, cuja redução foi de apenas 7,5\% entre 2013 e 2019 (DATASUS, 2021).

O Ministério da Saúde do Brasil (MS) estabelece diretrizes para um acompanhamento pré-natal efetivo, incluindo a realização de exames laboratoriais, procedimentos clínico-obstétricos, atividades educativas, imunização e orientações às mulheres e suas famílias (Brasil, 2000; Brasil, 2011; Brasil, 2016). Contudo, considerou-se, também, as atuais recomendações da Organização Mundial da Saúde (OMS) para possibilitar comparação com outros países que envolvem alguns procedimentos e intervenções além das recomendações do MS (WHO, 2016). 
A ausência de cumprimento dos protocolos na assistência pré-natal pode ser determinada por fatores pessoais, econômicos, comportamentos de risco, crenças e organizacionais dos serviços pré-natal (Nussey et al., 2020; Reis et al., 2015; Silva et al., 2019). Assim, o estudo objetiva classificar o atendimento pré-natal de mulheres atendidas para o parto em maternidades do Espírito Santo por meio de um índice universal, envolvendo as recomendações da Organização Mundial da Saúde (OMS) e um índice nacional, do Ministério da Saúde do Brasil (MS), além de analisar os fatores associados à inadequação do pré-natal.

\section{Metodologia}

Estudo transversal, cujos dados estaduais foram obtidos em maternidades do Espírito Santo, a partir de uma pesquisa nacional de base hospitalar, "Nascer no Brasil", realizada entre fevereiro de 2011 e outubro de 2012. A amostra foi selecionada em três estágios. No primeiro, incluiu-se hospitais com mais de 500 partos/ano, que foram estratificados segundo macrorregiões do país (Norte, Sul, Nordeste, Sudeste e Centro-oeste), localização (capital ou interior) e tipo de serviço (público, privado ou misto). No segundo estágio, definiu-se o número de dias necessários para entrevistar 90 puérperas em cada um dos 266 hospitais selecionados previamente (mínimo de 7 dias), utilizando o método de amostragem inversa. E no terceiro estágio, selecionou-se as puérperas e seus conceptos. Informações adicionais sobre o desenho amostral podem ser encontradas no estudo de Vasconcellos et al. (2014).

Para esta análise, especificamente, considerou-se apenas as puérperas que tiveram partos em cinco maternidades do estado do Espírito Santo. Embora não sejam representativas do estado, cobriram diferentes tipos de serviço (duas públicas, duas mistas e uma privada). Utilizou-se informações da entrevista com a puérpera durante a internação hospitalar por meio de um questionário eletrônico e dados do cartão pré-natal, que foram fotografados e transcritos para formulário padronizado. Maiores detalhes sobre a coleta de dados estão descritos em Leal et al. (2012).

A variável desfecho relaciona-se à adequação da assistência pré-natal segundo recomendações da OMS (WHO, 2016) e do MS (Brasil, 2000; Brasil, 2011; Brasil, 2016) e inclui informações retiradas prioritariamente do cartão da gestante. Ambas abordam suplementação de ferro e ácido fólico, exames de rotina (hemograma, cultura de urina, glicemia de jejum, teste HIV, VDRL), procedimentos clínicos obstétricos (movimentos fetais, palpação abdominal/ sínfise-altura uterina), realização de ultrassonografia (pelo menos uma), imunização antitetânica e número de consultas pré-natal (Brasil, 2000; Brasil, 2011; Brasil, 2016; WHO, 2016). Além desses itens, a recomendação da OMS preconiza verificar o uso de tabaco e de álcool e o recebimento do cartão da gestante (WHO, 2016).

A recomendação do MS aborda, adicionalmente, o início do pré-natal até a $12^{\mathrm{a}}$ semana, exames (ABO-Rh, EAS, HBsAg, Toxoplasmose, Anti-HCV, citopatológico de colo uterino), encaminhamento para maternidade, ausculta dos batimentos cardiofetais e verificação da pressão arterial materna (Brasil, 2000; Brasil, 2011; Brasil, 2016). Neste estudo, critérios avaliativos presentes na recomendação original, como alimentação saudável, atividade física, violência doméstica, atividades educativas em grupo não foram incluídos por não estarem contidos no instrumento da pesquisa.

Criou-se um índice que toma por base o referencial teórico de Donabedian (2003) e Silva et al. (2019), e tem como critérios avaliativos as recomendações dos órgãos de saúde nacionais e internacionais (MS e OMS). Quanto às recomendações da OMS e do MS, para cada componente da adequação pré-natal realizado a gestante somou um ponto, exceto para os exames de repetição e procedimentos clínico-obstétricos em toda consulta que somou dois pontos.

Posteriormente, classificou-se o pré-natal com base na porcentagem do número de itens adequados em relação ao número total de questões. A classificação ocorreu da seguinte forma: adequado superior, quando $100 \%$ dos itens encontravamse adequados; adequado, de 75\% a 99\% dos itens adequados; intermediário, de 51\% a 74\% dos itens adequados; e inadequado, $50 \%$ ou menos dos critérios avaliados em conformidade com as recomendações propostas. Para a análise inferencial, as 
categorias foram agrupadas em pré-natal adequado (adequado superior e adequado) e inadequado (intermediário e inadequado).

Outras variáveis incluídas no estudo foram: idade materna; raça/cor da pele; nível de escolaridade; classificação econômica segundo a Associação Brasileira de Institutos de Pesquisa de Mercado (2010) (A/B, C, D/E); situação conjugal; trabalho remunerado; mulher chefe da família; se queria engravidar naquele momento; se morava na capital ou em outros municípios; local do pré-natal (serviço público, privado ou ambos); paridade; aborto anterior; prematuridade anterior; baixo peso anterior e doenças gestacionais ou pré-gestacionais (anemia, infecção urinária, diabetes, sífilis e hipertensão).

Para verificar se as características socioeconômicas, do pré-natal, antecedentes obstétricos e doenças pré-gestacionais ou gestacionais apresentavam diferenças de proporções entre mulheres com pré-natal adequado (adequado superior e adequado) e inadequado (intermediário e inadequado) utilizou-se o teste qui-quadrado, com intervalo de confiança de $95 \%$. Posteriormente, realizou-se regressão logística univariada e múltipla para identificar os fatores associados à inadequação do pré-natal. Em todas as regressões foram testados os efeitos de interação antes da análise final (escolaridade versus escore socioeconômico, situação conjugal versus trabalho remunerado, escolaridade versus trabalho remunerado, escore socioeconômico versus local de moradia e paridade versus local do pré-natal), e quando presentes foram mantidos nas análises. $\mathrm{Na}$ análise múltipla foram incluídas todas as variáveis com $\mathrm{p}$-valor $<10 \%$ na análise univariada. Os modelos foram testados manualmente e escolheu-se com melhor ajuste aquele cujo valor da estatística pseudo-R2 (Cox \& Snell e Nagelkerke) estava mais próximo de 1 e o teste de Hosmer-Lemeshow apresentou p>0,05.

A pesquisa foi aprovada pelo Comitê de Ética em Pesquisa da Escola Nacional de Saúde Pública da Fundação Oswaldo Cruz, sob n ${ }^{\circ}$ 92/2010. Obteve-se o consentimento digital de cada puérpera após a leitura do termo de consentimento livre e esclarecido antes da entrevista.

\section{Resultados}

Das 23.894 puérperas que participaram da amostra "Nascer no Brasil" 450 tiveram seus partos realizados em hospitais do Espírito Santo. Destas, $447(99,3 \%)$ receberam assistência pré-natal e $431(95,8 \%)$ portavam o cartão da gestante. Entre aquelas com cartão, cerca de $70 \%(n=313)$ possuía-o durante a internação hospitalar para o parto.

Tabela 1. Componentes da adequação pré-natal segundo recomendações da Organização Mundial da Saúde e do Ministério da Saúde, Espírito Santo, 2011-2012.

\begin{tabular}{lccc}
\hline & Total & n & \% \\
\hline RECOMENDAÇÕES DA OMS & & & \\
\hline Intervenções nutricionais & & & \\
\hline Suplementação de ferro & 313 & 72 & 23,0 \\
Ácido fólico & 313 & 66 & 21,1 \\
\hline Avaliação Materna & & & \\
\hline Hemograma & 310 & 261 & 84,2 \\
Cultura de urina & 310 & 206 & 66,5 \\
Glicemia de jejum & 310 & 262 & 84,5 \\
Teste HIV & 310 & 242 & 78,1 \\
VDRL & 311 & 272 & 87,7 \\
Não usou tabaco & 313 & 278 & 88,8 \\
Não usou álcool/ não usou inadequadamente & 305 & 270 & 88,5 \\
\hline Avaliação do feto & & & \\
\hline Movimentos fetais & 313 & 275 & 87,9 \\
Palpação abdominal/ sínfise-altura uterina & 313 & 277 & 88,5 \\
Ultrassonografia (pelo menos uma) & 313 & 294 & 93,9 \\
Imunização antitetânica & 312 & 173 & 55,4
\end{tabular}


Research, Society and Development, v. 10, n. 12, e290101220375, 2021

(CC BY 4.0) | ISSN 2525-3409 | DOI: http://dx.doi.org/10.33448/rsd-v10i12.20375

\begin{tabular}{|c|c|c|c|}
\hline 8 consultas de pré-natal & 313 & 127 & 40,6 \\
\hline Cartão da gestante & 313 & 313 & 100 \\
\hline Classificação da adequação pré-natal OMS & 313 & & \\
\hline Adequado superior & & 14 & 4,5 \\
\hline Adequado & & 140 & 44,7 \\
\hline Intermediário & & 114 & 36,4 \\
\hline Inadequado & & 45 & 14,4 \\
\hline \multicolumn{4}{|l|}{ RECOMENDAÇÕ̃S DO MS } \\
\hline Início do pré-natal até a $12^{\mathrm{a}}$ semana & 308 & 130 & 42,2 \\
\hline Mínimo de 6 consultas & 313 & 217 & 69,3 \\
\hline $\mathrm{ABO}-\mathrm{Rh}$ & 311 & 278 & 89,4 \\
\hline VDRL & 311 & & \\
\hline Um & & 142 & 45,7 \\
\hline Dois & & 130 & 41,8 \\
\hline Teste HIV & 310 & & \\
\hline Um & & 164 & 52,9 \\
\hline Dois & & 78 & 25,2 \\
\hline Glicemia de jejum & 310 & & \\
\hline Um & & 114 & 36,8 \\
\hline Dois & & 148 & 47,7 \\
\hline Hemograma & 310 & & \\
\hline Um & & 121 & 39,0 \\
\hline Dois & & 140 & 45,2 \\
\hline Urina & 310 & & \\
\hline Um & & 106 & 34,2 \\
\hline Dois & & 129 & 41,6 \\
\hline HBsAg & 310 & & \\
\hline Um & & 171 & 55,2 \\
\hline Dois & & 84 & 27,1 \\
\hline Cultura de urina & 310 & 206 & 66,5 \\
\hline Toxoplasmose & 310 & 260 & 83,9 \\
\hline Anti-HCV & 309 & 80 & 25,9 \\
\hline Ultrassonografia (pelo menos uma) & 313 & 294 & 93,9 \\
\hline Citopatológico de colo uterino & 313 & 29 & 9,3 \\
\hline Imunização antitetânica & 312 & 173 & 55,4 \\
\hline Encaminhamento para maternidade & 313 & 182 & 58,1 \\
\hline Suplementação de ferro & 313 & 72 & 23,0 \\
\hline Suplementação de ácido fólico & 313 & 66 & 21,1 \\
\hline Movimentos fetais & 313 & & \\
\hline Em parte das consultas & & 22 & 7,0 \\
\hline Toda consulta & & 275 & 87,9 \\
\hline Palpação abdominal/sínfise-altura uterina & 313 & & \\
\hline Em parte das consultas & & 30 & 9,6 \\
\hline Toda consulta & & 277 & 88,5 \\
\hline Ausculta dos batimentos cárdio-fetais & 313 & & \\
\hline Em parte das consultas & & 21 & 6,7 \\
\hline Toda consulta & & 284 & 90,7 \\
\hline Verificação da pressão arterial & 313 & & \\
\hline Em parte das consultas & & 12 & 3,8 \\
\hline Toda consulta & & 300 & 95,8 \\
\hline Classificação da adequação pré-natal MS & 313 & & \\
\hline Adequado superior & & 1 & 0,3 \\
\hline Adequado & & 135 & 43,1 \\
\hline Intermediário & & 116 & 37,1 \\
\hline Inadequado & & 61 & 19,5 \\
\hline
\end{tabular}

Fonte: Autores. 
Quanto às recomendações da OMS, mais da metade dos itens obteve $80 \%$ ou mais de execução do protocolo previsto durante o pré-natal, enquanto a suplementação de ferro e ácido fólico foram os itens menos executados, cerca de $20 \%$. Já as recomendações do MS mostram que os procedimentos clínico-obstétricos foram os itens mais executados, ao passo que o exame citopatológico de colo uterino foi o menos registrado no cartão da gestante (Tabela 1).

Tabela 2. Caracterização das puérperas quanto à classificação da adequação do pré-natal segundo recomendações da Organização Mundial da Saúde, Espírito Santo, 2011-2012.

\begin{tabular}{|c|c|c|c|c|c|c|c|}
\hline & \multicolumn{2}{|c|}{$\begin{array}{c}\text { Todas as mulheres } \\
\mathrm{n}=313\end{array}$} & \multicolumn{2}{|c|}{$\begin{array}{c}\text { Pré-natal adequado } \\
\mathrm{n}=154(49,2 \%)\end{array}$} & \multicolumn{2}{|c|}{$\begin{array}{c}\text { Pré-natal inadequado } \\
n=159(50,8 \%)\end{array}$} & \multirow{2}{*}{$\begin{array}{c}\chi^{2} \\
\text { p-valor }\end{array}$} \\
\hline & $\mathbf{n}$ & $\%$ & $\mathbf{n}$ & $\%$ & $\mathbf{n}$ & $\%$ & \\
\hline Idade materna & & & & & & & 0,057 \\
\hline$\leq 19$ anos & 59 & 18,8 & 21 & 13,6 & 38 & 23,9 & \\
\hline 20 a 34 anos & 228 & 72,8 & 118 & 76,6 & 110 & 69,2 & \\
\hline$\geq 35$ anos & 26 & 8,3 & 15 & 9,7 & 11 & 6,9 & \\
\hline Raça/cor & & & & & & & 0,133 \\
\hline Branca & 65 & 20,8 & 35 & 22,7 & 30 & 18,9 & \\
\hline Preta & 53 & 16,9 & 19 & 12,3 & 34 & 21,4 & \\
\hline Parda & 191 & 61,0 & 97 & 63,0 & 94 & 59,1 & \\
\hline Outros & 4 & 1,3 & 3 & 1,9 & 1 & 0,6 & \\
\hline Escolaridade & & & & & & & $<0,001$ \\
\hline Fundamental incompleto & 87 & 27,9 & 25 & 16,2 & 62 & 39,2 & \\
\hline Fundamental completo & 88 & 28,2 & 47 & 30,5 & 41 & 25,9 & \\
\hline Médio completo & 119 & 38,1 & 68 & 44,2 & 51 & 32,3 & \\
\hline Superior e mais & 18 & 5,8 & 14 & 9,1 & 4 & 2,5 & \\
\hline Classificação socioeconômica & & & & & & & 0,016 \\
\hline Classe D+E (baixa) & 69 & 22,1 & 24 & 15,6 & 45 & 28,5 & \\
\hline Classe C (média) & 174 & 55,8 & 90 & 58,4 & 84 & 53,2 & \\
\hline Classe A+B (alta) & 69 & 22,1 & 40 & 26,0 & 29 & 18,4 & \\
\hline Situação conjugal & & & & & & & 0,002 \\
\hline Com companheiro & 257 & 82,1 & 137 & 89,0 & 120 & 75,5 & \\
\hline Sem companheiro & 56 & 17,9 & 17 & 11,0 & 39 & 24,5 & \\
\hline Trabalho remunerado & & & & & & & $<0,001$ \\
\hline Não & 181 & 57,8 & 72 & 46,8 & 109 & 68,6 & \\
\hline Sim & 132 & 42,2 & 82 & 53,2 & 50 & 31,4 & \\
\hline Mulher chefe da família & & & & & & & 0,203 \\
\hline Não & 282 & 90,7 & 142 & 92,8 & 140 & 88,6 & \\
\hline Sim & 29 & 9,3 & 11 & 7,2 & 18 & 11,4 & \\
\hline Intenção de engravidar & & & & & & & 0,002 \\
\hline $\begin{array}{l}\text { Queria engravidar naquele } \\
\text { momento }\end{array}$ & 125 & 40,2 & 76 & 49,4 & 49 & 31,2 & \\
\hline Queria esperar mais tempo & 67 & 21,5 & 32 & 20,8 & 35 & 22,3 & \\
\hline Não queria engravidar & 119 & 38,3 & 46 & 29,9 & 73 & 46,5 & \\
\hline Local de moradia & & & & & & & $<0,001$ \\
\hline Capital & 181 & 57,8 & 105 & 68,2 & 76 & 47,8 & \\
\hline Demais municípios & 132 & 42,2 & 49 & 31,8 & 83 & 52,2 & \\
\hline Local do pré-natal & & & & & & & $<0,001$ \\
\hline Privado & 72 & 23,1 & 48 & 31,2 & 24 & 15,2 & \\
\hline Público & 223 & 71,5 & 93 & 60,4 & 130 & 82,3 & \\
\hline Ambos & 17 & 5,4 & 13 & 8,4 & 4 & 2,5 & \\
\hline Paridade & & & & & & & 0,038 \\
\hline Primíparas & 126 & 40,3 & 71 & 46,1 & 55 & 34,6 & \\
\hline Multíparas & 187 & 59,7 & 83 & 53,9 & 104 & 65,4 & \\
\hline
\end{tabular}


Research, Society and Development, v. 10, n. 12, e290101220375, 2021

(CC BY 4.0) | ISSN 2525-3409 | DOI: http://dx.doi.org/10.33448/rsd-v10i12.20375

\begin{tabular}{|c|c|c|c|c|c|c|c|}
\hline Aborto anterior* & & & & & & & 0,135 \\
\hline Não & 137 & 69,5 & 66 & 75,0 & 71 & 65,1 & \\
\hline Sim & 60 & 30,5 & 22 & 25,0 & 38 & 34,9 & \\
\hline Prematuridade anterior* & & & & & & & 0,512 \\
\hline Não & 161 & 86,1 & 73 & 88,0 & 88 & 84,6 & \\
\hline Sim & 26 & 13,9 & 10 & 12,0 & 16 & 15,4 & \\
\hline Baixo peso anterior* & & & & & & & 0,068 \\
\hline Não & 159 & 85,0 & 75 & 90,4 & 84 & 80,8 & \\
\hline Sim & 28 & 15,0 & 8 & 9,6 & 20 & 19,2 & \\
\hline Anemia $(n=254)$ & & & & & & & 0,469 \\
\hline Não & 187 & 73,6 & 110 & 75,3 & 77 & 71,3 & \\
\hline Sim & 67 & 26,4 & 36 & 24,7 & 31 & 28,7 & \\
\hline Infecção urinária $(\mathrm{n}=258)$ & & & & & & & 0,894 \\
\hline Não & 183 & 70,9 & 109 & 71,2 & 74 & 70,5 & \\
\hline Sim & 75 & 29,1 & 44 & 28,8 & 31 & 29,5 & \\
\hline Diabetes $(n=262)$ & & & & & & & 0,567 \\
\hline Não & 232 & 88,5 & 132 & 87,4 & 100 & 90,1 & \\
\hline Sim & 5 & 1,9 & 4 & 2,6 & 1 & 0,9 & \\
\hline Em risco & 25 & 9,5 & 15 & 9,9 & 10 & 9,0 & \\
\hline Sífilis (n=272) & & & & & & & 0,581 \\
\hline Não & 269 & 98,9 & 153 & 99,4 & 116 & 98,3 & \\
\hline Sim & 3 & 1,1 & 1 & 0,6 & 2 & 1,7 & \\
\hline Hipertensão $(n=313)$ & & & & & & & 0,424 \\
\hline Não & 260 & 83,1 & 124 & 80,5 & 136 & 85,5 & \\
\hline Gestacional & 37 & 11,8 & 20 & 13,0 & 17 & 10,7 & \\
\hline Prévia & 16 & 5,1 & 10 & 6,5 & 6 & 3,8 & \\
\hline
\end{tabular}

Fonte: Autores.

As puérperas com maior inadequação do pré-natal segundo as recomendações da OMS foram as adolescentes, com ensino fundamental incompleto, de classe econômica D+E (baixa), sem companheiro, sem trabalho remunerado, que não queriam engravidar, residiam fora da capital, realizaram o pré-natal no setor público e eram multíparas (Tabela 2). Após ajustar o modelo final permaneceram associadas à inadequação do pré-natal não ter trabalho remunerado, não querer engravidar, residir fora da capital do estado, realizar o pré-natal no setor público e ser multípara (Tabela 3). 
Tabela 3. Regressão logística ajustada dos fatores associados à inadequação do pré-natal segundo recomendações da Organização Mundial da Saúde, Espírito Santo, 2011-2012.

\begin{tabular}{|c|c|c|c|c|}
\hline & OR bruta & IC95\% & OR ajustada & IC $95 \%$ \\
\hline \multicolumn{5}{|l|}{ Idade materna } \\
\hline$\leq 19$ anos & 1,94 & $1,07-3,51$ & - & - \\
\hline 20 a 34 anos & 1,00 & - & - & - \\
\hline$\geq 35$ anos & 0,57 & $0,35-1,79$ & & \\
\hline \multicolumn{5}{|l|}{ Escolaridade } \\
\hline Fundamental incompleto & 8,68 & $2,60-28,94$ & - & - \\
\hline Fundamental completo & 3,05 & $0,93-10,01$ & - & - \\
\hline Médio completo & 2,62 & $0,82-8,44$ & - & - \\
\hline Superior e mais & 1,00 & - & - & - \\
\hline \multicolumn{5}{|l|}{ Classificação socioeconômica } \\
\hline Classe D+E (baixa) & 2,59 & $1,30-5,15$ & - & - \\
\hline Classe C (média) & 1,29 & $0,73-2,26$ & - & - \\
\hline Classe A+B (alta) & 1,00 & - & - & - \\
\hline \multicolumn{5}{|l|}{ Situação conjugal } \\
\hline Com companheiro & 1,00 & - & - & - \\
\hline Sem companheiro & 2,62 & $1,41-4,87$ & - & - \\
\hline \multicolumn{5}{|l|}{ Trabalho remunerado } \\
\hline Não & 2,48 & $1,57-3,94$ & 2,09 & $1,22-3,57$ \\
\hline Sim & 1,00 & - & 1,00 & - \\
\hline \multicolumn{5}{|l|}{ Intenção de engravidar } \\
\hline Queria engravidar naquele momento & 1,00 & - & 1,00 & - \\
\hline Queria esperar mais tempo & 1,70 & $0,93-3,09$ & 1,17 & $0,60-2,27$ \\
\hline Não queria engravidar & 2,46 & $1,47-4,11$ & 1,91 & $1,08-3,37$ \\
\hline \multicolumn{5}{|l|}{ Local de moradia } \\
\hline Capital & 1,00 & - & 1,00 & - \\
\hline Demais municípios & 2,34 & $1,48-3,71$ & 1,87 & $1,11-3,17$ \\
\hline \multicolumn{5}{|l|}{ Local do pré-natal } \\
\hline Privado & 1,00 & - & 1,00 & - \\
\hline Público & 2,80 & $1,60-4,88$ & 4,92 & $1,65-14,68$ \\
\hline Ambos & 0,62 & $0,18-2,09$ & 2,60 & $0,32-20,98$ \\
\hline \multicolumn{5}{|l|}{ Paridade } \\
\hline Primíparas & 1,00 & - & 1,00 & - \\
\hline Multíparas & 1,62 & $1,03-2,55$ & 9,85 & $2,92-33,14$ \\
\hline Público*Multípara & & & 0,11 & $0,29-0,42$ \\
\hline Ambos*Multípara & & & 0,05 & $0,004-0,75$ \\
\hline \multicolumn{5}{|l|}{ Baixo peso anterior } \\
\hline Não & 1,00 & - & - & - \\
\hline Sim & 2,23 & $0,93-5,37$ & - & - \\
\hline
\end{tabular}

Fonte: Autores.

$\mathrm{Na}$ Tabela 4 são expostas as puérperas com maior inadequação do pré-natal segundo as recomendações do MS, que são mulheres com ensino fundamental incompleto, de classe econômica baixa $(\mathrm{D}+\mathrm{E})$, que não queriam engravidar e que residiam fora da capital. Após ajuste do modelo permaneceram associadas à inadequação do pré-natal aquelas que não queriam engravidar e que residiam fora da capital do estado (Tabela 5). 
Tabela 4. Caracterização das puérperas quanto à classificação da adequação do pré-natal segundo recomendações do Ministério da Saúde, Espírito Santo, 2011-2012.

\begin{tabular}{|c|c|c|c|c|c|c|c|}
\hline & \multicolumn{2}{|c|}{$\begin{array}{c}\text { Todas as mulheres } \\
\mathrm{n}=313\end{array}$} & \multicolumn{2}{|c|}{$\begin{array}{c}\text { Pré-natal adequado } \\
n=136(\%)\end{array}$} & \multicolumn{2}{|c|}{$\begin{array}{c}\text { Pré-natal inadequado } \\
\mathrm{n}=177(\%)\end{array}$} & \multirow{2}{*}{$\begin{array}{c}\chi^{2} \\
\text { p-valor }\end{array}$} \\
\hline & $\mathbf{n}$ & $\%$ & $\mathbf{n}$ & $\%$ & $\mathbf{N}$ & $\%$ & \\
\hline Idade materna & & & & & & & 0,175 \\
\hline$\leq 19$ anos & 59 & 18,8 & 20 & 14,7 & 39 & 22,0 & \\
\hline 20 a 34 anos & 228 & 72,8 & 102 & 75,0 & 126 & 71,2 & \\
\hline$\geq 35$ anos & 26 & 8,3 & 14 & 10,3 & 12 & 6,8 & \\
\hline Raça/cor & & & & & & & 0,397 \\
\hline Branca & 65 & 20,8 & 25 & 18,4 & 40 & 22,6 & \\
\hline Preta & 53 & 16,9 & 20 & 14,7 & 33 & 18,6 & \\
\hline Parda & 191 & 61,0 & 90 & 66,2 & 101 & 57,1 & \\
\hline Outros & 4 & 1,3 & 1 & 0,7 & 3 & 1,7 & \\
\hline Escolaridade & & & & & & & 0,047 \\
\hline Fundamental incompleto & 87 & 27,9 & 27 & 19,9 & 60 & 34,1 & \\
\hline Fundamental completo & 88 & 28,2 & 42 & 30,9 & 46 & 26,1 & \\
\hline Médio completo & 119 & 38,1 & 59 & 43,4 & 60 & 34,1 & \\
\hline Superior e mais & 18 & 5,8 & 8 & 5,9 & 10 & 5,7 & \\
\hline Classificação socioeconômica & & & & & & & 0,056 \\
\hline Classe D+E (baixa) & 69 & 22,1 & 23 & 16,9 & 46 & 26,1 & \\
\hline Classe C (média) & 174 & 55,8 & 76 & 55,9 & 98 & 55,7 & \\
\hline Classe A+B (alta) & 69 & 22,1 & 37 & 27,2 & 32 & 18,2 & \\
\hline Situação conjugal & & & & & & & 0,692 \\
\hline Com companheiro & 257 & 82,1 & 113 & 83,1 & 144 & 81,4 & \\
\hline Sem companheiro & 56 & 17,9 & 23 & 16,9 & 33 & 18,6 & \\
\hline Trabalho remunerado & & & & & & & 0,283 \\
\hline Não & 181 & 57,8 & 74 & 54,4 & 107 & 60,5 & \\
\hline Sim & 132 & 42,2 & 62 & 45,6 & 70 & 39,5 & \\
\hline Mulher chefe da família & & & & & & & 0,158 \\
\hline Não & 282 & 90,7 & 126 & 93,3 & 156 & 88,6 & \\
\hline Sim & 29 & 9,3 & 9 & 6,7 & 20 & 11,4 & \\
\hline Intenção de engravidar & & & & & & & 0,008 \\
\hline $\begin{array}{l}\text { Queria engravidar naquele } \\
\text { momento }\end{array}$ & 125 & 40,2 & 67 & 50,0 & 58 & 32,8 & \\
\hline Queria esperar mais tempo & 67 & 21,5 & 23 & 17,2 & 44 & 24,9 & \\
\hline Não queria engravidar & 119 & 38,3 & 44 & 32,8 & 75 & 42,4 & \\
\hline Local de moradia & & & & & & & 0,004 \\
\hline Capital & 181 & 57,8 & 91 & 66,9 & 90 & 50,8 & \\
\hline Demais municípios & 132 & 42,2 & 45 & 33,1 & 87 & 49,2 & \\
\hline Local do pré-natal & & & & & & & 0,700 \\
\hline Privado & 72 & 23,1 & 30 & 22,1 & 42 & 23,9 & \\
\hline Público & 223 & 71,5 & 97 & 71,3 & 126 & 71,6 & \\
\hline Ambos & 17 & 5,4 & 9 & 6,6 & 8 & 4,5 & \\
\hline Paridade & & & & & & & 0,600 \\
\hline Primíparas & 126 & 40,3 & 57 & 41,9 & 69 & 39,0 & \\
\hline Multíparas & 187 & 59,7 & 79 & 58,1 & 108 & 61,0 & \\
\hline Aborto anterior* & & & & & & & 0,930 \\
\hline Não & 137 & 69,5 & 58 & 69,9 & 79 & 69,3 & \\
\hline Sim & 60 & 30,5 & 25 & 30,1 & 35 & 30,7 & \\
\hline Prematuridade anterior* & & & & & & & 0,396 \\
\hline Não & 161 & 86,1 & 70 & 88,6 & 91 & 84,3 & \\
\hline Sim & 26 & 13,9 & 9 & 11,4 & 17 & 15,7 & \\
\hline
\end{tabular}


Research, Society and Development, v. 10, n. 12, e290101220375, 2021

(CC BY 4.0) | ISSN 2525-3409 | DOI: http://dx.doi.org/10.33448/rsd-v10i12.20375

\begin{tabular}{|c|c|c|c|c|c|c|c|}
\hline Baixo peso anterior* & & & & & & & 0,448 \\
\hline Não & 159 & 85,0 & 69 & 87,3 & 90 & 83,3 & \\
\hline Sim & 28 & 15,0 & 10 & 12,7 & 18 & 16,7 & \\
\hline Anemia $(n=254)$ & & & & & & & 0,184 \\
\hline Não & 187 & 73,6 & 94 & 70,1 & 93 & 77,5 & \\
\hline Sim & 67 & 26,4 & 40 & 29,9 & 27 & 22,5 & \\
\hline Infecção urinária (n=258) & & & & & & & 0,076 \\
\hline Não & 183 & 70,9 & 90 & 66,2 & 93 & 76,2 & \\
\hline Sim & 75 & 29,1 & 46 & 33,8 & 29 & 23,8 & \\
\hline Diabetes $(n=262)$ & & & & & & & 0,397 \\
\hline Não & 232 & 88,5 & 118 & 86,8 & 114 & 90,5 & \\
\hline Sim & 5 & 1,9 & 4 & 2,9 & 1 & 0,8 & \\
\hline Em risco & 25 & 9,5 & 14 & 10,3 & 11 & 8,7 & \\
\hline Súfilis $(\mathrm{n}=272)$ & & & & & & & 0,553 \\
\hline Não & 269 & 98,9 & 133 & 98,5 & 136 & 99,3 & \\
\hline Sim & 3 & 1,1 & 2 & 1,5 & 1 & 0,7 & \\
\hline Hipertensão $(\mathrm{n}=313)$ & & & & & & & 0,173 \\
\hline Não & 260 & 83,1 & 107 & 78,7 & 153 & 86,4 & \\
\hline Gestacional & 37 & 11,8 & 21 & 15,4 & 16 & 9,0 & \\
\hline Prévia & 16 & 5,1 & 8 & 5,9 & 8 & 4,5 & \\
\hline
\end{tabular}

Fonte: Autores.

Tabela 5. Regressão logística ajustada dos fatores associados à inadequação do pré-natal segundo recomendações do Ministério da Saúde, Espírito Santo, 2011-2012.

\begin{tabular}{|c|c|c|c|c|}
\hline & OR bruta & IC95\% & OR ajustada & IC $95 \%$ \\
\hline \multicolumn{5}{|l|}{ Escolaridade } \\
\hline Fundamental incompleto & 1,78 & $0,63-5,00$ & - & - \\
\hline Fundamental completo & 0,88 & $0,32-2,43$ & - & - \\
\hline Médio completo & 0,81 & $0,30-2,20$ & - & - \\
\hline Superior e mais & 1,00 & - & - & - \\
\hline \multicolumn{5}{|l|}{ Classificação socioeconômica } \\
\hline Classe D+E (baixa) & 2,31 & $1,16-4,61$ & - & - \\
\hline Classe C (média) & 1,49 & $0,85-2,61$ & - & - \\
\hline Classe A+B (alta) & 1,00 & - & - & - \\
\hline \multicolumn{5}{|l|}{ Intenção de engravidar } \\
\hline $\begin{array}{l}\text { Queria engravidar naquele } \\
\text { momento }\end{array}$ & 1,00 & - & 1,00 & - \\
\hline Queria esperar mais tempo & 2,21 & $1,19-4,09$ & 2,00 & $1,07-3,75$ \\
\hline Não queria engravidar & 1,97 & $1,18-3,29$ & 1,88 & $1,12-3,16$ \\
\hline \multicolumn{5}{|l|}{ Local de moradia } \\
\hline Capital & 1,00 & - & 1,00 & - \\
\hline Demais municípios & 1,96 & $1,23-3,11$ & 1,91 & $1,17-3,08$ \\
\hline \multicolumn{5}{|l|}{ Infecção urinária } \\
\hline Não & 1,64 & $0,95-2,83$ & - & - \\
\hline Sim & 1,00 & - & - & - \\
\hline
\end{tabular}

Fonte: Autores.

\section{Discussão}

Mais da metade das puérperas estudadas no Espírito Santo tiveram o pré-natal classificado como inadequado, sendo a falta de trabalho remunerado, não querer engravidar, residir fora da capital do estado, realizar o pré-natal no setor público e ser 
multípara os fatores associados. Quanto ao protocolo da OMS, acima de 50\% dos itens obtiveram 80\% ou mais de execução, sendo a suplementação de ferro e ácido fólico os menos executados. Com base nas recomendações do MS, os procedimentos clínico-obstétricos foram os itens mais efetuados e o exame citopatológico de colo uterino o menos registrado no cartão da gestante.

Apesar de serem recomendações antigas, observa-se a ausência da realização de ações mínimas preconizadas pelo Programa de Humanização no Pré-natal e Nascimento - PHPN (Guimarães et al., 2018; Mario et al., 2019) e pela OMS. Os achados sugerem que a atenção pré-natal do ES não incorporou adequadamente a imunização antitetânica e a suplementação de ferro e ácido fólico à rotina do serviço, indo de encontro aos resultados encontrados em estudos que também analisaram o cartão de pré-natal em Aracaju e João Pessoa (Alves da Silva Carvalho et al., 2016; Silva et al., 2019), e que se basearam em entrevista com gestantes (Cesar et al., 2011; Tomasi et al., 2017).

Por outro lado, a baixa realização do exame citopatológico de colo uterino assemelha-se aos achados de outros estudos (Alves da Silva Carvalho et al., 2016; Tomasi et al., 2017), o que mostra a oportunidade perdida pelo serviço, isto é, por deixar de examinar mulheres que podem não estar sendo acompanhadas rotineiramente para rastrear lesões cancerizáveis ou cancerígenas (Terlan \& Cesar, 2018).

A não execução desses procedimentos e exames no setor público pode ser consequência de dificuldades encontradas na organização dos serviços de saúde, incluindo falta de materiais e insumos nas unidades de saúde para vacinação, suplementação e realização de exames, não funcionamento de equipamentos e dificuldades de agendamento dos exames (Polgliane et al., 2014). Enquanto no setor privado, a ausência de esclarecimento por parte dos profissionais de saúde sobre a importância da vacinação e suplementação pode levar à baixa adesão da gestante, já que esses procedimentos ocorrem fora do ambiente de acompanhamento pré-natal.

Nota-se ainda uma grande iniquidade na oferta dos cuidados pré-natais, principalmente relacionadas à raça/cor da pele e às classes econômicas (Guimarães et al., 2018; Mario et al., 2019; Osterman \& Joyce A. Martin, 2018). A falta de cumprimento de quesitos básicos da atenção à gestante é um desafio em saúde pública que necessita de maior investimento, de aprimoramento da assistência e da ampliação do acesso no âmbito da Estratégia de Saúde da Família - ESF, pois a adequada estruturação e oferta impactam positivamente na redução da morbimortalidade materna e infantil (Guimarães et al., 2018; Mario et al., 2019).

A desvantagem econômica refletida pela ausência do trabalho remunerado diminui o poder aquisitivo das famílias, expondo e reduzindo as opções da gestante ao acesso aos cuidados de saúde. Essa condição, por vezes, pode retardar ou impedir o ingresso da mulher aos serviços de saúde e ao acompanhamento contínuo do pré-natal, ocasionando problemas durante a gestação, parto e puerpério. Estudos anteriores, que analisaram a saúde materna em situação de recessões econômicas, demonstraram que a taxa de desemprego está associada de forma negativa ao momento de entrada no atendimento pré-natal e ao peso ao nascer e, contudo, se associa positivamente à mortalidade infantil (Blakeney et al., 2019; Catalano et al., 2011).

Mulheres que receberam a assistência ao pré-natal no setor público apresentaram maior chance de tê-lo de forma inadequada. Possivelmente, como única via de cuidados, o SUS se torna a opção acessível às necessidades de saúde da maioria da população. Entretanto devido aos problemas acarretados pelo subfinanciamento e organização do sistema, alguns serviços de saúde não possuem equipes e equipamentos mínimos necessários para execução do pré-natal ou mesmo insumos terapêuticos como medicamentos, vacinas e suplementos (Polgliane et al., 2014; Silva et al., 2019).

Além disso, mais de 70\% dos atendimentos à gestante ocorrem no âmbito do SUS, que atende mulheres com perfis de saúde mais complexos, grandes multíparas, adolescentes, com baixo nível socioeconômico, com mais comorbidades, entre 
outros, que requerem uma rede de cuidados que supra, em todos os níveis, a assistência necessária para evitar possíveis desfechos negativos maternos e perinatais (Cesar et al., 2011; Silva et al., 2019).

Residir fora da capital, outro fator associado à assistência inadequada ao pré-natal, aponta o efeito direto da concentração de recursos e tecnologias em algumas regiões de saúde (Landim et al., 2019). Apesar do pré-natal ser um serviço prioritariamente da atenção primária, ainda existem iniquidades e desigualdades intra e inter-regionais nas redes de atenção à saúde, evidenciando a presença de maiores barreiras de acesso em cenários menos desenvolvidos, longe dos grandes centros (Landim et al., 2019), revelado pela falta de exames, medicamentos, vacinas, suplementos e até mesmo profissionais de saúde.

Mulheres que não desejavam engravidar e as multíparas tiveram mais chances de apresentar pré-natal inadequado. A literatura aponta que mulheres com gravidez não intencionada postergam mais o início do acompanhamento e realizam menor número de consultas e exames necessários (Rocha et al., 2017; Rosa et al., 2014). Quando a gestação não é intencional pode despertar o sentimento de negação, afetando a qualidade do acompanhamento pré-natal (Theme-Filha et al., 2016). Quanto às multíparas sem complicações obstétricas prévias, o atraso da busca por assistência à saúde aumenta em função da experiência adquirida, além da dificuldade de deixar os filhos com alguém enquanto realiza o acompanhamento pré-natal (Rocha et al., 2017; Rosa et al., 2014).

Multíparas com histórico de prematuridade e complicações em gestações anteriores apresentam maior chance de gestação não planejada, assim como mulheres que usam tabaco e álcool. Essas mulheres com comportamento de risco normalmente não pretendem gestar, portanto, são menos cientes da importância de melhorar hábitos, em comparação às mulheres com uma gestação planejada, podendo resultar em desfechos negativos para a mãe e o feto (Theme-Filha et al., 2016).

Apesar da maioria dos procedimentos ter um percentual de execução elevado, ainda há desigualdade nos cuidados oferecidos às gestantes, as mulheres mais vulnerabilizadas foram as que mais tiveram inadequação do pré-natal, obedecendo à lei dos cuidados inversos (Rasia \& Albernaz, 2008), o que demanda um papel mais social dos serviços públicos de saúde. Entretanto, deve-se destacar que, em relação às recomendações do MS, as desigualdades foram minimizadas, fato bastante positivo.

Ao comparar as duas recomendações, percebe-se que a da OMS é menos abrangente que a do MS, pois suas recomendações atendem à população mundial, incluindo países de baixa renda. A maior amplitude das recomendações e a execução do pré-natal conforme as recomendações nacionais podem ter contribuído para a diminuição das iniquidades entre as gestantes, já que apenas não querer engravidar e morar fora da capital contribuíram para a inadequação do pré-natal.

Entretanto realizar a análise segundo recomendações internacionais possibilita a análise do desempenho do Brasil comparado aos outros países. Uma revisão sistemática (Morón-Duarte et al., 2019), que descreveu os indicadores utilizados para avaliar a qualidade do atendimento pré-natal segundo as recomendações da OMS, mostrou que na maioria dos continentes as ações mais pesquisadas são exames de rotina, procedimentos clínico-obstétricos, imunização antitetânica e promoção de comportamentos relacionados à saúde materna e neonatal.

Este estudo destaca-se por utilizar duas classificações da adequação do pré-natal, uma nacional e outra internacional, por analisar partos em hospitais públicos, privados e mistos, por levar em consideração mais do que a idade gestacional no início do pré-natal e o número de consultas realizadas. Todavia apresenta limitações como o fato de as recomendações da OMS serem posteriores à coleta de dados, embora a maioria dos itens que compõem as recomendações já fossem preconizadas pelo MS, contendo campo para registro no cartão da gestante, além de servirem para verificar em que medida as ações de pré-natal já vinham sendo executadas na época da coleta de dados. Outra limitação é a não representatividade amostral das puérperas analisadas, além da não inclusão de hospitais com menos de 500 partos por ano, que representavam 22,9\% dos nascimentos para o ano de 2007 (Vasconcellos et al., 2014). 


\section{Considerações Finais}

Procedimentos clínico-obstétricos, exames sorológicos e ultrassonográficos são ações bem consolidadas no atendimento pré-natal, entretanto intervenções nutricionais, repetição dos exames sorológicos, captação precoce das gestantes, exame citopatológico de colo uterino e vacinação antitetânica ainda apresentam baixos percentuais de execução ou registro no cartão da gestante, especialmente em mulheres mais vulneráveis. Essa realidade não se justifica, pois são procedimentos de baixo custo há muito preconizados pelo MS. Ampliar o acesso de todas as mulheres a um pré-natal em conformidade com as diretrizes atuais depende tanto da redução das iniquidades sociais quanto da reorganização dos serviços de saúde, ampliando o acesso da população desde o planejamento familiar até exames, vacinas, suplementos e medicamentos em quantidade e tempo oportunos, para que se possa evitar possíveis desfechos negativos maternos e perinatais.

Novas pesquisas devem ser realizadas ao longo dos anos para que possamos avaliar a evolução da atenção as mulheres e seus recém-nascidos, principalmente utilizando as novas recomendações da OMS, para que a atenção pré-natal do Brasil possa ser comparada internacionalmente.

\section{Agradecimentos}

Os autores agradecem a todos os envolvidos na realização da pesquisa "Nascer no Brasil", incluindo coordenadores regionais e nacionais, coordenadores de coleta de dados, coordenadores de unidades, coletores de dados e equipe das unidades participantes.

\section{Referências}

Alves, da S. C., R., Santana Santos, V., Moura de Melo, C., Queiroz Gurgel, R., \& Costa da Cunha Oliveira, C. (2016). Avaliação da adequação do cuidado pré-natal segundo a renda familiar em Aracaju, 2011. Epidemiologia e Serviços de Saúde, 25(2), 1-2. https://doi.org/10.5123/S1679-49742016000200006.

Associação Brasileira de Empresas de Pesquisa (2010). Critério de classificação econômica Brasil. http://www.abep.org.

Blakeney, E. L., Herting, J. R., Bekemeier, B., \& Zierler, B. K. (2019). Social determinants of health and disparities in prenatal care utilization during the Great Recession period 2005-2010. BMC Pregnancy and Childbirth, 19(1), 390. https://doi.org/10.1186/s12884-019-2486-1.

Catalano, R., Goldman-Mellor, S., Saxton, K., Margerison-Zilko, C., Subbaraman, M., LeWinn, K., \& Anderson, E. (2011). The Health Effects of Economic Decline. Annual Review of Public Health, 32(1), 431-450. https://doi.org/10.1146/annurev-publhealth-031210-101146.

Cesar, J. A., Mano, P. S., Carlotto, K., Gonzalez-Chica, D. A., \& Mendoza-Sassi, R. A. (2011). Público versus privado: Avaliando a assistência à gestação e ao parto no extremo sul do Brasil. Revista Brasileira de Saúde Materno Infantil, 11(3), 257-263. https://doi.org/10.1590/S1519-38292011000300006.

Departamento de Informática do Sistema Único de Saúde (DATASUS) (2021). Estatísticas Vitais. BRASIL. http://www2.datasus.gov.br/DATASUS/index.php?area=0205.

Donabedian A (2003). An introduction to quality assurance in health care. New York: Oxford University Press.

Guimarães, W. S. G., Parente, R. C. P., Guimarães, T. L. F., \& Garnelo, L. (2018). Acesso e qualidade da atenção pré-natal na Estratégia Saúde da Família: Infraestrutura, cuidado e gestão. Cadernos de Saúde Pública, 34(5). https://doi.org/10.1590/0102-311x00110417.

Heaman, M. I., Martens, P. J., Brownell, M. D., Chartier, M. J., Derksen, S. A., \& Helewa, M. E. (2019). The Association of Inadequate and Intensive Prenatal Care With Maternal, Fetal, and Infant Outcomes: A Population-Based Study in Manitoba, Canada. Journal of Obstetrics and Gynaecology Canada, 41(7), 947-959. https://doi.org/10.1016/j.jogc.2018.09.006.

Landim, E. L. A. S., Guimarães, M. do C. L., \& Pereira, A. P. C. de M. (2019). Rede de Atenção à Saúde: Integração sistêmica sob a perspectiva da macrogestão. Saúde em Debate, 43(spe5), 161-173. https://doi.org/10.1590/0103-11042019s514.

Leal, M.C, da Silva, A. A. M., Dias, M. A. B., da Gama, S. G. N., Rattner, D., Moreira, M. E., Filha, M. M. T., Domingues, R. M. S. M., Pereira, A. P. E., Torres, J. A., \& others. (2012). Birth in Brazil: National survey into labour and birth. Reproductive Health, 9(1), 15.

Mario, D. N., Rigo, L., Boclin, K. de L. S., Malvestio, L. M. M., Anziliero, D., Horta, B. L., Wehrmeister, F. C., \& Martínez-Mesa, J. (2019). Qualidade do Pré-Natal no Brasil: Pesquisa Nacional de Saúde 2013. Ciência \& Saúde Coletiva, 24(3), 1223-1232. https://doi.org/10.1590/1413-81232018243.13122017.

Ministério da Saúde (BRASIL), Secretaria de Políticas de Saúde (2000). Programa de Humanização no Pré-natal e Nascimento. Brasília, DF.

Ministério da Saúde (BRASIL) (2016). Protocolos da atenção básica: saúde das mulheres. Brasília, DF: Ministério da Saúde; Instituto Sírio Libanês de Ensino e Pesquisa. 
Ministério da Saúde (BRASIL) (2011). Portaria consolidada Rede Cegonha Brasil. Brasília, DF. https://bvsms.saude.gov.br/bvs/saudelegis/gm/2011/prt1459_24_06_2011.html.

Morón-Duarte, L. S., Ramirez Varela, A., Segura, O., \& Freitas da Silveira, M. (2019). Quality assessment indicators in antenatal care worldwide: A systematic review. International Journal for Quality in Health Care, 31(7), 497-505. https://doi.org/10.1093/intqhc/mzy206.

Nussey, L., Hunter, A., Krueger, S., Malhi, R., Giglia, L., Seigel, S., Simpson, S., Wasser, R., Patel, T., Small, D., \& Darling, E. K. (2020). Sociodemographic Characteristics and Clinical Outcomes of People Receiving Inadequate Prenatal Care: A Retrospective Cohort Study. Journal of Obstetrics and Gynaecology Canada, 42(5), 591-600. https://doi.org/10.1016/j.jogc.2019.08.005.

Osterman, M. J. K. \& Joyce A. Martin. (2018). Timing and Adequacy of Prenatal Care in the United States, 2016.14.

Polgliane, R. B. S., Leal, M. do C., Amorim, M. H. C., Zandonade, E., \& Santos Neto, E. T. dos. (2014). Adequação do processo de assistência pré-natal segundo critérios do Programa de Humanização do Pré-natal e Nascimento e da Organização Mundial de Saúde. Ciência \& Saúde Coletiva, 19(7), 1999-2010. https://doi.org/10.1590/1413-81232014197.08622013.

Rasia, I. C. R. B., \& Albernaz, E. (2008). Atenção pré-natal na cidade de Pelotas, Rio Grande do Sul, Brasil. Revista Brasileira de Saúde Materno Infantil, 8(4), 401-410. https://doi.org/10.1590/S1519-38292008000400005.

Reis, P. A. da G. D. dos, Pereira, C. C. de A., Leite, I. da C., \& Theme Filha, M. M. (2015). Fatores associados à adequação do cuidado pré-natal e à assistência ao parto em São Tomé e Príncipe, 2008-2009. Cadernos de Saúde Pública, 31(9), 1929-1940. https://doi.org/10.1590/0102-311X00115914.

Rocha, I. M. da S., Barbosa, V. S. de S., \& Lima, A. L. da S. (2017). Fatores que influenciam a não adesão ao programa de pré-natal. Revista Recien - Revista Científica de Enfermagem, 7(21), 21. https://doi.org/10.24276/rrecien2358-3088.2017.7.21.21-29.

Rosa, C. Q. da, Silveira, D. S. da, \& Costa, J. S. D. da. (2014). Factors associated with lack of prenatal care in a large municipality. Revista de Saúde Pública, 48(6), 977-984. https://doi.org/10.1590/S0034-8910.2014048005283.

Santos Neto, E. T. dos, Oliveira, A. E., Zandonade, E., \& Leal, M. do C. (2013). Access to prenatal care: Assessment of the adequacy of different indices. Cadernos de Saúde Pública, 29(8), 1664-1674. https://doi.org/10.1590/S0102-311X2013001200018.

Silva, E. P. da, Leite, A. F. B., Lima, R. T., \& Osório, M. M. (2019). Prenatal evaluation in primary care in Northeast Brazil. Revista de Saúde Pública, 53, 43. https://doi.org/10.11606/S1518-8787.2019053001024.

Terlan, R. J., \& Cesar, J. A. (2018). Não realização de citopatológico de colo uterino entre gestantes no extremo sul do Brasil: Prevalência e fatores associados. Ciência \& Saúde Coletiva, 23(11), 3557-3566. https://doi.org/10.1590/1413-812320182311.35162016.

Theme-Filha, M. M., Baldisserotto, M. L., Fraga, A. C. S. A., Ayers, S., da Gama, S. G. N., \& Leal, M. do C. (2016). Factors associated with unintended pregnancy in Brazil: Cross-sectional results from the Birth in Brazil National Survey, 2011/2012. Reproductive Health, 13(S3), 118. https://doi.org/10.1186/s12978-016-0227-8.

Tomasi, E., Fernandes, P. A. A., Fischer, T., Siqueira, F. C. V., Silveira, D. S. da, Thumé, E., Duro, S. M. S., Saes, M. de O., Nunes, B. P., Fassa, A. G., \& Facchini, L. A. (2017). Qualidade da atenção pré-natal na rede básica de saúde do Brasil: Indicadores e desigualdades sociais. Cadernos de Saúde Pública, 33(3). https://doi.org/10.1590/0102-311x00195815.

Vale, C. C. R., Almeida, N. K. de O., \& Almeida, R. M. V. R. de. (2021). Association between Prenatal Care Adequacy Indexes and Low Birth Weight Outcome. RBGO Gynecology and Obstetrics, 43(04), 256-263. https://doi.org/10.1055/s-0041-1728779.

Vasconcellos, M. T. L. de, Silva, P. L. do N., Pereira, A. P. E., Schilithz, A. O. C., Souza Junior, P. R. B. de, \& Szwarcwald, C. L. (2014). Desenho da amostra Nascer no Brasil: Pesquisa Nacional sobre Parto e Nascimento. Cadernos de Saúde Pública, 30, S49-S58. https://doi.org/10.1590/0102-311X00176013.

Yeoh, P. L., Hornetz, K., Shauki, N. I. A., \& Dahlui, M. (2018). Evaluating the quality of antenatal care and pregnancy outcomes using content and utilization assessment. International Journal for Quality in Health Care, 30(6), 466-471. https://doi.org/10.1093/intqhc/mzy041.

World Health Organization (WHO) (2016). Recommendations on antenatal care for a positive pregnancy experience. Geneva: World Health Organization. https://www.ncbi.nlm.nih.gov/books/NBK409108/. 